DE

\title{
REVELATIONE RELIGIONIS
}

\author{
EXTERNA EADEMQUE \\ P U B L I C A \\ PROLUSIONES ACADEMICAE.
}

S C R I P I T

RECOGNOVIT ET EMENDAVIT

\section{CARolts Ludovicus Nitzsch,}

THEOLOGIAE PROFESSOR IN ACAD. VITEBERG. ETC.

VITEBERGENSIS CIRCULI SUPERINTENDENS

GENERALIS.

\section{$\begin{array}{lllllll}L & \text { I } & \text { P } & \text { S } & 1 & \text { A } & \text { E }\end{array}$}

APUd G. J. GÖSCHENT. CIOTJCCCVIII. 
